\title{
The Description of Reservoiring Model for Gas Hydrate Based on the Sparse Spike Inversion
}

\author{
Qingshan Zhang, Ruizhao Yang, Lingbin Meng, Tian Zhang, Pengpeng Li \\ School of Geosciences \& Surveying Engineering \\ China University of Mining \& Technology, Beijing \\ Beijing, China \\ zhqsh121@126.com
}

\begin{abstract}
Considering the charateristics of marine gas hydrate containing area such as faulted structures are developed and reservoir distribution is not clear, this paper uses the recursive inversion method based on deconvolution to carry out the poststack constrained sparse spike inversion without well data by integrating the well log responses of gas hydrates in adjacent areas. The inversion result has been sculptured in order to find out the gas hydrate forming pattern and characterize the spatial distribution of gas hydrates and further predict the favorable area containing gas hydrates. The research result shows that the research area has obvious gas chimney responses, and the deep gas take gas chimneys as their migration pathways and migrate all the way to somewhre in the top to form gas hydrate reservoirs, which can provide practical reference and basis for future exploration and production of marine gas hydrates.
\end{abstract}

Keywords-gas hydrate; constraint sparse pulse inversion; reservoiring model; gas chimney

\section{INTRODUCTION}

Gas hydrate have been recognized internationally because of its characteristics of huge resources, large energy density, convenient to use, high combustion value, clean pollution-free, and became the clean energy which would be developed firstly and Vigorously in various countries ${ }^{[1]}$. The reserves of gas hydrate more than 2 times that of the resources of existing combustible mineral, and regional distribution is very extensive (90\% of the sea and $27 \%$ of the land), has been list national as an important goal of future energy countermeasures ${ }^{[2]}$. According to the calculation, the resources of gas hydrate in the south China sea is 70 billion tons of oil equivalent, about a half of the total resources of oil and gas onshore in China ${ }^{[3]}$.

The research situation of gas hydrate domestic: (1) started late relatively, but developed rapidly; (2) arisen from the laboratory studies, and then extended to resources survey areas; (3) the research about ocean gas hydrate is more active than about permafrost; (4) the research about gas hydrates domestic is obvious professional, and led the world in production, storage and transportation of gas hydrate ${ }^{[4]}$, but not enough in the research and application of geophysical exploration, and geological aspects mainly refer to overseas achievements and experience, lack of original theory ${ }^{[5]}$.

Because of work area without drilling and logging data, we can only evaluated and predicted gas hydrate reservoir based on seismic data in this area. This article combined the techniques of post-stack without well constrained sparse spike inversion and gas chimney detecting, realized the prediction of the favorable zones for the distribution of gas hydrate. Analyzed the difference and distribution of gas hydrate impedance values, to summarize the accumulation pattern of gas hydrate in this area, obtained a good effect, which provide a reference for the quantitative forecast and mining of seas gas hydrates afterwards.

\section{THEORY AND METHOD}

Sparse spike inversion (SSI) is a method of seismic impedance inversion was major used currently [6]. Constrained sparse spike inversion is a kind of deterministic inversion method, its essence is a recursive inversion method based on the deconvolution arithmetic ${ }^{[7]}$.

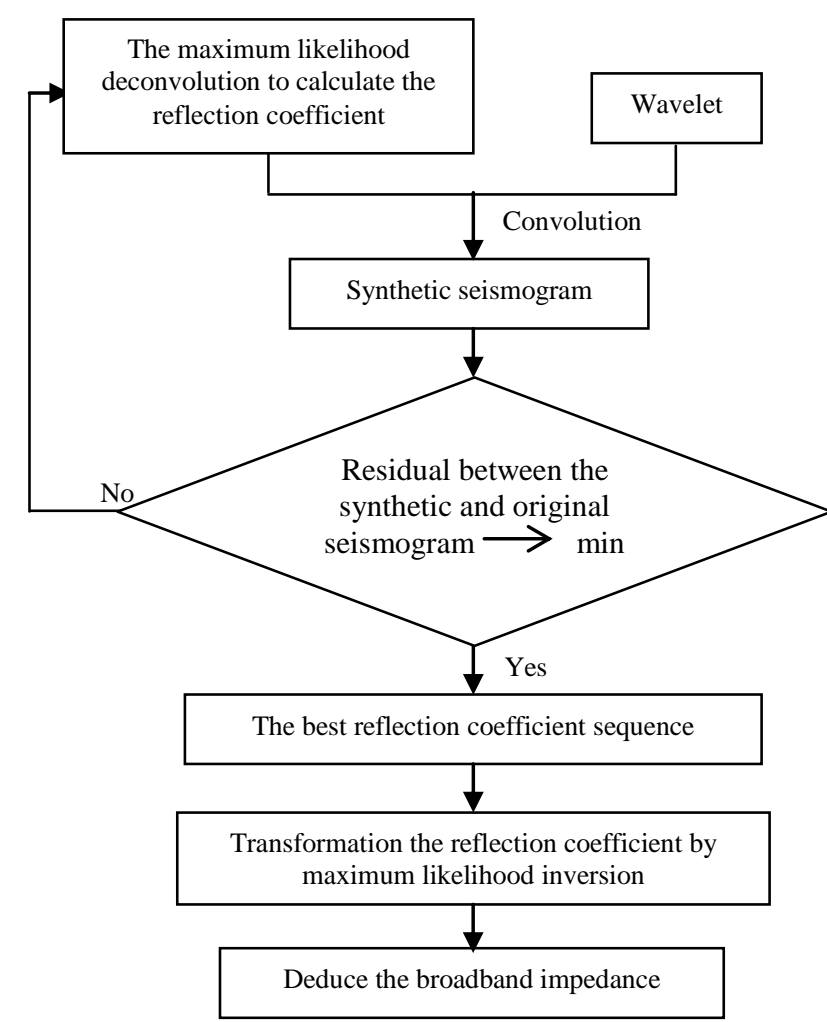

Fig. 1 Flow diagram of sparse pulse inversion 
Sparse spike inversion method can understand like that the convolution between seismic wavelet in the trace with sparse principle and reflection coefficient was synthesized into seismic record, modified the reflection coefficient according to residual between the synthetic and original seismogram, get a new synthetic seismogram from the next convolution, then estimated this new residual, continued above process until the residuals was small enough ${ }^{[8]}$. Loop iteration got a synthetic seismic records most close to the original seismic trace. As shown in fig. 1 .

The impedance result from constrained sparse pulse inversion composed of three parts of frequency source: Low frequency $(0 \sim 8 \mathrm{HZ})$ from low frequency model; Intermediate frequency $(8 \sim 60 \mathrm{HZ})$ from seismic; And High frequency (55 65HZ) from constrained sparse pulse inversion algorithm. The inversion method is mainly divided into the following three steps:

\section{A. Use Maximum Likelihood Deconvolution to Obtain Sparse Reflection Coefficient Sequence}

The maximum likelihood deconvolution is used to inverse the reflection coefficient, and the minimum objective function is derived as follows:

$$
J=\frac{1}{R^{2}} \sum_{K=1}^{L} r^{2}(K)+\frac{1}{N^{2}} \sum_{K=1}^{L} n^{2}(K)-2 M \ln (\lambda)-2(L-M) \ln (1-\lambda)
$$

In the formula, $\mathrm{R}^{2}$ and $\mathrm{N}^{2}$ are the mean square values of the reflection coefficient and the noise. $r(K)$ and $n(K)$ represent the reflection coefficient and the noise of the Kth sampling point, $M$ represents the reflection layer, $L$ represents the total number of samples, $\lambda$ indicates the likelihood value of a given reflection coefficient.

According to this objective function, the position of the reflection coefficient is assumed, and the magnitude of the reflection coefficient is judged. After repeated iteration, the position and amplitude of the corresponding reflection coefficient are modified, until the error meets the criterion of the minimum likelihood ratio, that is, complete a deconvolution, the distribution of the reflection coefficient is obtained.

\section{B. Base On The Inversion Results of Reflection Coefficient, Calculate An initial Broadband Impedance.}

If $r(i)$ is the reflection coefficient obtained in the maximum likelihood deconvolution, the impedance is

$$
Z(i)=Z(i-1) \frac{1+r(i)}{1-r(i)}
$$

In the formula, $\mathrm{Z}(\mathrm{i})$ for the impedance of the ith layer, $r(i)$ for the reflection coefficient of the ith layer.

\section{Wave Impedance Inversion Under constraint Conditions}

Constrained sparse pulse inversion of each adjusted according to the initial wave impedance to calculate the objective function, including the reflection coefficient adjustment. The optimal objective function as the minimum calculated error function is:

$$
\text { objf }=\sum\left|r_{i}\right|^{p}+\lambda^{q}\left(d_{i}-s\right)^{q}+\alpha^{2} \sum\left(t_{i}-z_{i}\right)^{2}
$$

In the formula, objf is for the objective function; $r_{i}$ is for the reflection coefficient, $\lambda$ is for seismic matching coefficient; $\mathrm{d}_{i}$ is for the seismic data; $s$ is for the synthesis of seismic data; $\alpha$ is trend matching coefficient; $t_{i}$ is for user defined trend; $z_{i}$ is for wave impedance control by user defined, The default value of $p=1, q=2$. Specifically, the first is the approximate linear sum of absolute value of reflection coefficient, the second is the sum of the variance of original seismic trace and synthetic seismic, the third is the compensation of the user defined trends and impedance coordination trend.

\section{APPLICATION EXAMPLE}

\section{A. Build Low Frequency Model}

There is not directly basis to build framework model and the low frequency model not well as no well in this area.

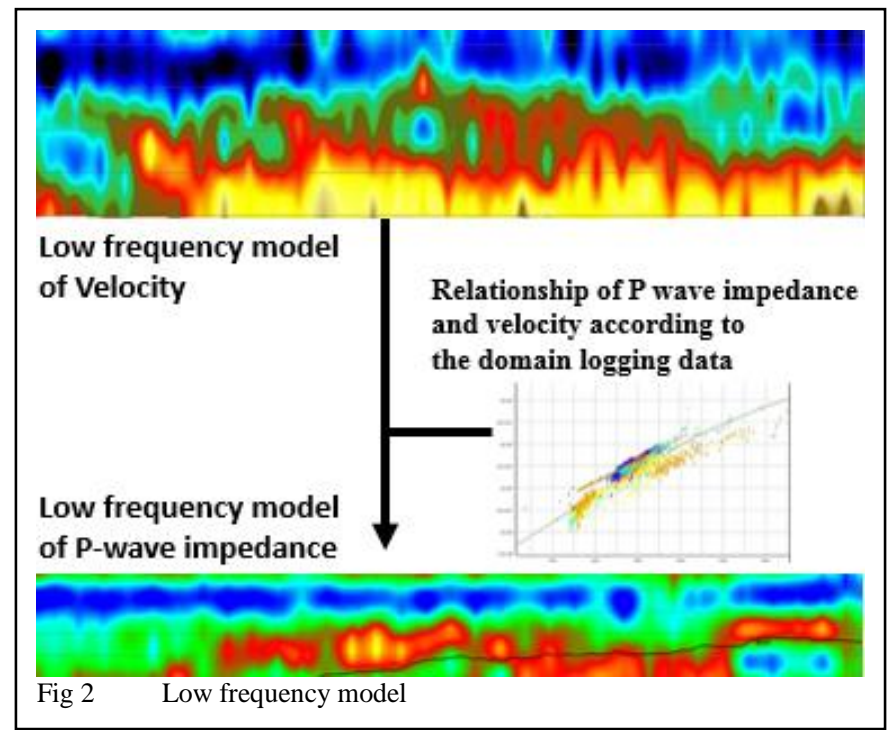

The crux is how to effectively establish a relatively accurate low frequency wave impedance model with using the existing stacking velocity data. The Velmod module of Jason

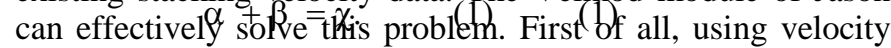
spectrum data to establish 3D velocity field of whole area to acquire seismic stack velocity information for any point, then converting stacking velocity to interval velocity. Finally, smoothing or filtering the interval velocity, and establishing an interval velocity field accord with geological characteristic, using the interval velocity after interpolating to establish data 
grid interval and velocity data volume in accordance with the seismic data grid. The mainly process of velocity spectrum treatment includes interval velocity transformation and velocity spectrum correction.

Getting a reasonable low frequency velocity model through the disposal and optimization of velocity spectrum, then using the logging data in domain and predecessors studying results, combining the digital rock physics analysis, obtaining a more accurate linear relationship between P-wave velocity and impedance, finally, using the relationship fitting $p$-wave impedance low frequency model, as shown in figure 2.

\section{B. Wavelet Extraction}

Seismic wavelet is a component of seismic convolution model, usually is a seismic pulse consisted of two or three or more phase, to be exact, the seismic wavelet is the far field response of particle move velocity (using land geophone) or pressure (using marine geophone) recorded when the seismic energy transmitted to receiver through the complex underground path from the source in time domain. A wavelet can be defined by its amplitude and phase spectrum. The types of phase spectrum can be zero phase, constant phase, minimum phase or mixed phase, etc. For the wavelet of zero phase and constant phase, it can be simple considered as a series of different amplitude and frequency sine wave, all of the sine wave are zero phase or constant phase (such as 90 degree); In the frequency domain, wavelet extraction consists of two parts: determine the amplitude spectrum and the phase spectrum. It more difficult to determine the phase spectrum, and it is the main source of error in inversion.

Merely extract the statistical wavelet using seismic data as no logging data in this area. The range of extraction is controlled in $300 \mathrm{~ms}$ below the seabed, as shown in figure 3 .

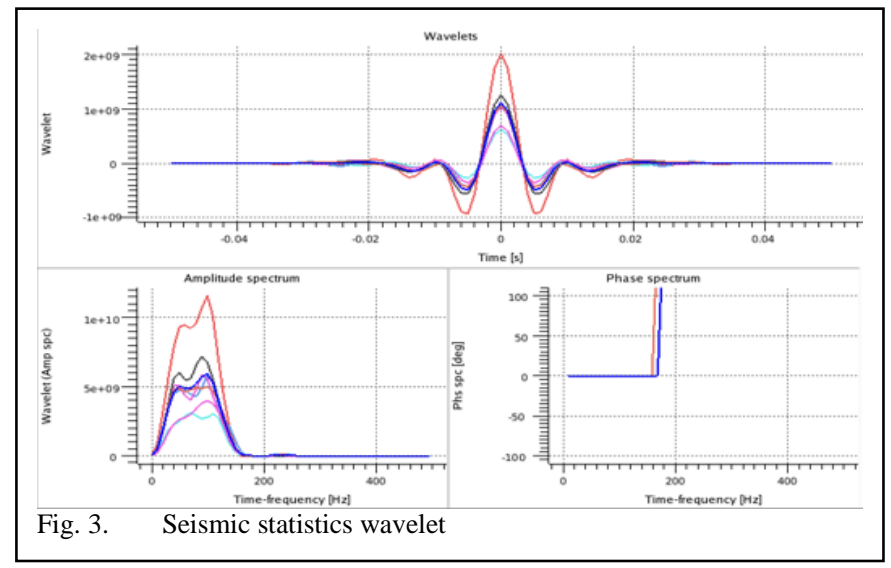

\section{Inversion Results}

The core step of the sparse spike inversion for impedance is the establishment of the relative impedance cube, it means that using the amplitude of seismic trace to establish the impedance model. It can be realized by the Constrained Sparse Spike functions of InverTrace module in Jason. The most critical thing is the choice of $\lambda$ (the sparse spike control factor). The value of $\lambda$ reflect the impedance values and the match degree between synthetic and the actual seismic trace. Through the experiment, the $\lambda$ value is 15 in this time. As shown in figure 4 is a inversion profile of $\mathrm{P}$ wave impedance for a certain line in work area.

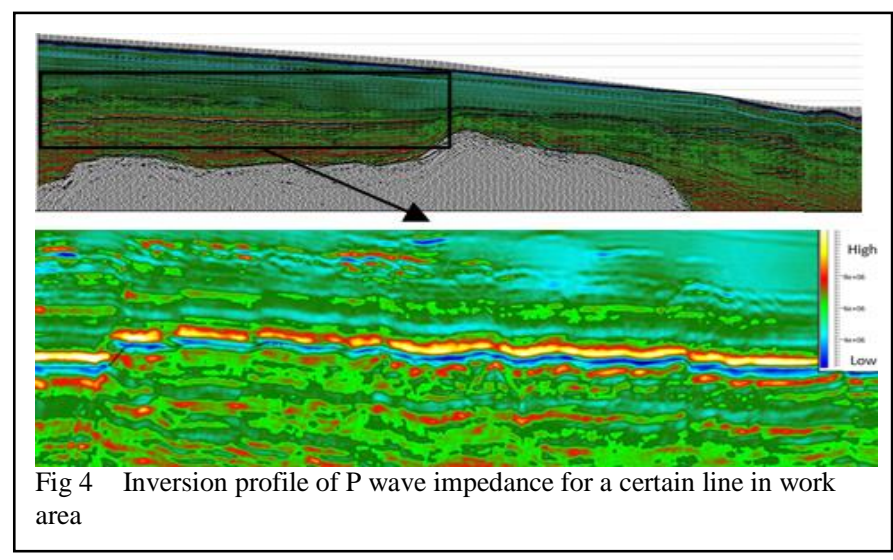

\section{Reservoiring Model Description}

In oil-gas geology, gas chimney is the channel of hydrocarbon migration and the indication sign of oil-gas migrate pattern ${ }^{[9]}$, once gas chimney formed, it will become an important channel for migration of oil-gas, and reveal the oilgas characteristic such as form site, migrate channel, and the reservoir location $^{[10]}$.

The migrate condition is superior the faulted structure develops well in target area, the faulted structure provides a good channel for the source of biogas underlie the stability gas hydrate and pyrolysis gas in depth to migrate to shallow stability area of gas hydrate. The source gas form into gas hydrate when the condition of stability domain is suitable.

Carved the inversion result, can more clearly show the gas chimney structure in the process of deep gas migrate upward. As shown in figure 5 , the gas chimney is given priority to migration channel in the study area, BSR is mainly distributed at the top of and around gas chimney, the fracture occurred in the process of gas chimney formation provide channels for the upward migration of methane-rich fluid, the fluid turn into gas hydrate in the slumping bloc on the top of gas chimney, then constituted a set of complete system for accumulation.

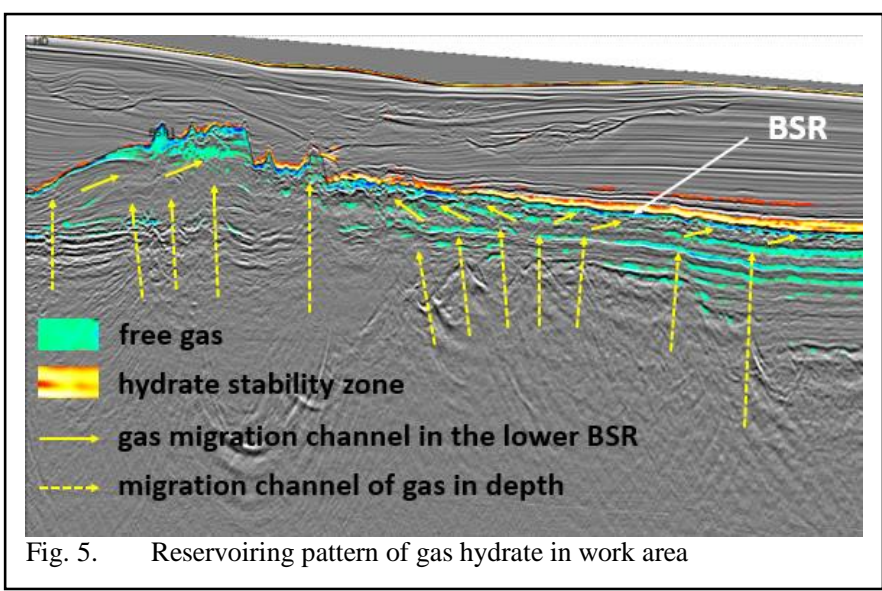




\section{CONCLUSIONS}

- The response of gas hydrate in seismic is abnormity of speed and amplitude (such as the characteristics of BSR, blank reflection).

- According to the logging information in domain, purely explain the growth of gas hydrate in p-wave impedance result in some of multiple solution, if there is elastic data, multiple solution can be reduced in some degree.

- Post-stack sparse spike inversion can get high quality Pwave impedance data volume, the existence of gas hydrate can be explained and depicted through the results of poststack sparse spike inversion.

- Preliminary judgment of the analysis by $\mathrm{P}$ impedance characteristics in work area, the northern, central and southern of target sea area developed abnormity of high P impedance, the abnormity is supposed to the favorable area for the development of gas hydrate.

- The gas chimney structure is developed in work area, the mainly reservoiring model is free gas in depth migrate upward along the gas chimney to the lower part of BSR, then migrate lateral, and accumulate in favorable areas.

\section{ACKNOWLEDGMENT}

We are appreciate for financial supports from Natural gas hydrate resources exploration and exploration project (GZH2011003-05-08-01) and China University of Mining and Technology in Beijing. Thanks for the platform of the 7th International Conference on Environmental and Engineering Geophysics support by Chinese Geophysical Society and
Institute of Geology and Geophysics Chinese Academy of Sciences.

\section{REFERENCES}

[1] Miller J. J. Myung W. L. VonHueneR, 1991, Ananaly sisofareflection from the base of a gas hydrate zone of Peru: American Association of Petroleum Geologists Bulletin, 75 V910-V924.

[2] Kalachand Sain, 2008, Seismic Methods for Recognition and Evaluation of Gas-Hydrates: IACMAG, 1, no. 6, V2189-V2196.

[3] Ojha, M. and Sain, K., 2007, Seismic amplitude versus angle (AVA) responses from a bottom simulating reflector of various gas-hydrates models: Geohorizons, 12, no. 2, V22-V26.

[4] Defu Yu, Huiling Chen. 2014, Our waters "combustible ice" investigation and exploration technology system: Chinese Territory Resource News, 01, no. 17, V001.

[5] Xuemei Lu, 2014, Big popular after shale gas: gas hydrates: China Petrochemical News, 02, no. 14, V008.

[6] Jin Li, 2012, Research on seismic inversion in the Second Member of Shahejie Formation-Dongying Formation of Wen'an slope Baxian Depression, M.S. thesis. Wu Han: Yangtze University.

[7] Ning Xu, 2007, Seismic characteristics of gas hydrates in east China sea and study of full waveform inversion M.S. thesis, Qing Dao: Institute of Oceanology, Chinese Academy of Sciences.

[8] Rui Yang, Yuan Zhang, Xinhua Lei, etc, 2011, Identification of gas hydrate reservoir and its depth estimation in Shenhu area South China Sea: Marine Geology \& Quaternary Geology, 31, no. 4. V141-V147.

[9] Zhibin Sha, Jinqiang Liang, Lifeng Wang, Zenggui Kuang, 2010, Application of gas chimney identification technique to study of the gas hydrates: The South China Sea geological research, no. 00, V7-V17.

[10] Xiujuan Wang, Shiguo WU, Dongdong Dong, Yuehua Dong, etc, 2008, Characteristics of gas chimney and its relationship to gas hydrate in Qiongdongnan basin: Marine Geology \& Quaternary Geology, 28, no. 03: V103-V108. 